Z Gerontol Geriat 2010 · 43:75-76 DOI 10.1007/s00391-009-0058-0

Online publiziert: 16. April 2010

๑) Springer-Verlag 2010

\section{T. Klie}

AGP - Alter.Gesellschaft.Partizipation, Institut für angewandte Sozialforschung im FIVE - Forschungs- und Innovationsverbund an der Evangelischen Hochschule Freiburg e. V.

\title{
Alter und Kommune
}

\section{Gestaltung des demographischen Wandels auf kommunaler Ebene}

Der demographische Wandel verändert das Gesicht von Städten und Gemeinden. Der Anteil älterer Menschen steigt (fast) überall - mal deutlich, mal moderat - sublokal, d. h. auf Quartiers- und Gemeindeebene mit teilweise sehr spezifischen Entwicklungen und Geschwindigkeiten. Während die einen Städte und Gemeinden noch für die nächsten Jahre mit Bevölkerungswachstum rechnen (können), steht anderen ein deutlicher Bevölkerungsrückgang bevor - z. T. in Kontinuität mit schon lang andauernden „Schrumpfungsprozessen“. Die zunehmende Zahl (hoch-)betagter Menschen schafft neue Anforderungen an die Infrastruktur, an die Alltagsversorgung, an die Wohnungswirtschaft und den Straßenverkehr. Unter dem Motto der ,age-friendly city" wurden die neuen Anforderungen an Städte und Gemeinden im demographischen Wandel formuliert. Die Gestaltungsaufgaben, die sich der Kommunalpolitik stellen, sind vielfältig und spezifisch. Patentrezepte lassen sich kaum formulieren. Ältere, insbesondere hochbetagte Menschen leben mehr „on the place“ und weniger „in space“. So kommt der Gestaltung von Wohnbedingungen, von Wohnumfeldern, von Quartieren und Dörfern und Dorfteilen für die Fragen der Lebensqualität, aber auch der Versorgung älterer Menschen große Bedeutung zu.

Die Paradigmen einer kommunalen Alterssozialpolitik haben sich geändert. Das macht Naegele in seinem Beitrag deutlich. Es ist nicht mehr Altenhilfe gemäß $₫ 71$ SGB XII, die als programmatische Vorga- be für eine moderne kommunale Altenplanung gelten kann. Die Gestaltung des demographischen Wandels unter besonderer Berücksichtigung der Potenziale, aber auch der Versorgungsbedarfe älterer Menschen ist eine Querschnittsaufgabe, die durch alle kommunalen Ressorts hindurchgeht und darüber hinaus die Wohnungswirtschaft ebenso wie Handel und Handwerk sowie die pflegerische Infrastruktur betrifft.

Böhme u. Franke stellen die Befunde zur Stadtentwicklungsplanung zum Thema Alter vor und zeigen damit auch, wie wichtig es für die Gerontologie ist, den Kontakt etwa zur Urbanistik, zur Stadtund Sozialplanung zu suchen und umgekehrt.

Auch die Themen zur „kommunalen Altenplanung" haben sich geändert: Es geht mitnichten allein um Versorgung, um die Anpassung der Infrastruktur, um altengerechte Gestaltung von Verkehrsund Konsumbereichen. Es geht um die Mitgestaltung, aber auch um die Mitverantwortung älterer Menschen mit Blick auf die Herausforderungen, die sich aus dem demographischen Wandel ergeben: Ältere Menschen sind im Planungsprozess als Bürgerinnen und Bürger, als bürgerschaftlich Engagierte sowie als Experten gefragt. Diese Mitgestaltungsrollen gehen über die in den letzten Jahrzehnten entstandenen Seniorenvertretungen hinaus. Seniorenvertretungen können gleichwohl im Rahmen von kommunaler Altenplanung wichtige Aufgaben übernehmen, wie etwa Piorkowsky in seinem Beitrag in diesem Heft illustriert.

\section{Es geht um Mitgestaltung und Mitverantwortung älterer Menschen}

„Fürs Alter planen“ - in dieser Verschränkung von individueller und kollektiver Vorsorge für das (eigene) Alter und ältere Menschen lässt sich ein moderner Planungsansatz abbilden. Ältere Menschen sind in ihrer individuellen Lebensplanung und -gestaltung herausgefordert, sich zentralen Fragen der sozialen Teilhabe, des Wohnens, der Sorge und Gesundheit zu stellen, und sind hier mit vielfältigen $\mathrm{Op}$ tionen ihrer Lebensgestaltung konfrontiert. Um diese Optionen zu erhalten bzw. möglichst vielen älteren Menschen zu öffnen, ergeben sich für die Kommunalpolitik Handlungsaufträge in vielfältiger Hinsicht. Dabei haben sich in den letzten Jahren die Vorzeichen für die Kommunalpolitik und die Sozialplanung geändert: Im Bereich der Pflege etwa ist eine klassische Bedarfsplanung mit Richtwerten für Pflegeheime und Pflegedienste seit Einführung der Pflegeversicherung, aber auch unter europarechtlichen Vorzeichen obsolet geworden. Diesen Fragen gehen Klie u. Pfundstein in ihrem Beitrag nach. Sie zeigen auf, dass neue Instrumente der Einflussnahme auf die Infrastrukturentwicklung in der Pflege gefragt sind und bislang in Betracht kommende Instrumente, etwa die Verträglichkeitsprüfungen im Bauplanungsrecht, kaum genutzt werden. 
Das Geld ist knapp. So bleiben im Wesentlichen an Governance orientierte Handlungsansätze, die auf Vernetzung, auf Moderation, auf Gewährleistung, aber nicht auf Gewährung ausgerichtet sind. Es lassen sich vielfältige Werkstätten interessanter koproduktiver Kultur- und Infrastrukturentwicklung auf kommunaler Ebene identifizieren. Die Sensibilität und das Problembewusstsein auf kommunalpolitischer Ebene wachsen langsam. Eine Reihe von Bundesländern haben Monitoringsysteme für die jeweils spezifischen demographischen Entwicklungstrends für die Kommunen entwickelt. Wie spezifisch sich die Probleme des demographischen Wandels gerade in den Kommunen der neuen Bundesländer zeigen, machen Dienel u. von Blanckenburg in ihrem Beitrag deutlich. Hier stellen sich sehr spezifische Fragen und ist Stadtentwicklung nicht selten mit Aufgaben des Rückbaus, der Renaturierung und einem produktiven Umgang mit Bevölkerungsrückgang konfrontiert: Das passt nicht in klassische wachstumsorientierte Planungstraditionen. Mit dem demographischen Wandel ist auch ein kultureller Wandel und Entwicklungsprozess verbunden. Altersbilder gilt es auch auf kommunaler Ebene, etwa in Leitbildern zum Thema "Älter werden“, ebenso weiterzuentwickeln und zu differenzieren wie das Marketing von Städten und Gemeinden. Manche Städte und Gemeinden haben sich bereits darauf eingestellt, ihre Attraktivität auch und gerade für ältere Menschen zu profilieren.

\section{( Kommunale Altenpolitik ist immer auch Generationenpolitik}

Mit dem Bau eines Altenheims, von Einrichtungen des Betreuten Wohnens oder einer Pflegeeinrichtung lässt sich heute das Anforderungsprofil der kommunalpolitischen Befassung mit dem Thema "Alter" nicht mehr adäquat beantworten. Mit Baudenkmälern allein lässt sich keine nachhaltige kommunale Altenpolitik machen, die immer auch eine Generationenpolitik ist: keine Infrastruktur für Ältere ohne Angebote für Kinder? Man wird darüber nachdenken müssen, wie man die Kommunen, die insbesondere durch die Pflegeversicherung in ihrer kommunalpolitischen Bedeutung beim Thema "Alter und Pflege“ marginalisiert wurden und ihre Regiekompetenz verloren haben, wieder mit neuen Steuerungskompetenzen und Ressourcen ausstattet. Die Aufgaben der Gestaltung des demographischen Wandels, sie liegt zweifellos bei den Kommunen, die Ressourcen und Kompetenzen bislang nicht. Alle kommunalpolitischen Aktivitäten, die sich in besonderer Weise (auch) auf ältere Menschen richten - viele kommen Familien mit Kindern und Menschen mit Behinderung ebenso zugute - und sich allein auf den Aufgabenbezug der Daseinsvorsorge stützen müssen, geraten in Zeiten der Finanznot der Kommunen strukturell in die Defensive.

\section{Korrespondenzadresse \\ Prof. Dr. T. Klie}

AGP - Alter.Gesellschaft.Partizipation, Institut für angewandte Sozialforschung im FIVE Forschungs- und Innovationsverbund an der Evangelischen Hochschule Freiburg e. V., Bugginger Str. 38, 79114 Freiburg klie@eh-freiburg.de 